\title{
Measuring Berry curvature with quantum Monte Carlo
}

\author{
Michael Kolodrubetz \\ Physics Department, Boston University, 590 Commonwealth Ave., Boston, MA 02215
}

\begin{abstract}
The Berry curvature and its descendant, the Berry phase, play an important role in quantum mechanics. They can be used to understand the Aharonov-Bohm effect, define topological Chern numbers, and generally to investigate the geometric properties of a quantum ground state manifold. While Berry curvature has been well-studied in the regimes of few-body physics and non-interacting particles, its use in the regime of strong interactions is hindered by the lack of numerical methods to solve it. In this paper we fill this gap by implementing a quantum Monte Carlo method to solve for the Berry curvature, based on interpreting Berry curvature as a leading correction to imaginary time ramps. We demonstrate our algorithm using the transverse-field Ising model in one and two dimensions, the latter of which is non-integrable. Despite the fact that the Berry curvature gives information about the phase of the wave function, we show that our algorithm has no sign or phase problem for standard sign-problem-free Hamiltonians. Our algorithm scales similarly to conventional methods as a function of system size and energy gap, and therefore should prove a valuable tool in investigating the quantum geometry of many-body systems.
\end{abstract}

From the Aharonov-Bohm effect [1, 2] to topological insulators $[3]$, geometry and topology play a major role in modern condensed matter physics. Topological properties of such systems yield edge states, quantized transport, and other robust physical quantities [1, 4, 5, 9, 13]. Topologically non-trivial systems have even been proposed as having major implications for cosmology [14].

Nearly all of the topological invariants in quantum mechanical systems are based on the concept of geometrical phase, a.k.a. Berry phase, a quantity that reflects the geometry of the ground state manifold [1. Berry phase is directly tied to a local tensor known as the Berry curvature. Integrals of the Berry curvature define many important topological quantities such as the Chern number [9, 11] and its presence in electron systems plays a role in the anomalous quantum Hall effect [15] and crystal polarization [16, 17].

While the Berry curvature and its integrals are relatively well understood for non-interacting electrons [4, 9, 10, 13, their use in strongly interacting systems remains in its infancy. For weakly-correlated systems, density functional theory does very well [16, but for strongly-correlated systems the best exact method to determine the Chern number is currently numerical diagonalization [17, 18, which scales very poorly with system size. Therefore, it is important to develop methods to extend calculations of the Berry curvature to larger system sizes.

In this paper, we develop a quantum Monte Carlo (QMC) method for measuring the (many-body) Berry curvature. QMC methods remain the gold standard for exact numerical methods in many-body physics, scaling efficiently with system size for a wide class of problems [19 21. Here we described and implement such a method which, similar to the original work by Berry [1, uses spin systems; in particular, we demonstrate our ideas using the quantum spin- $1 / 2$ transverse-field Ising (TFI) model in $d$ dimensions [22 26], which is a non-integrable strongly-interacting spin system for $d \geq 2$. The algorithm can be readily extended to other sign-problem-free spin [27-29], bosonic 30 32, or even certain fermionic systems 33, 34].

Given that the Berry curvature is a measure of the invariant ground state phase, it is surprising that QMC methods can solve this quantity without encountering the notorious sign problem. To accomplish this, we rewrite the Berry curvature as the leading-order correction to an asymmetric ramp in imaginary time [26, 35, whose dynamics can be solved exactly using sign-free QMC methods. At the end of the paper, we show that this algorithm scales with system size $L$ and gap $\Delta$ comparably to ground state algorithms for more conventional observables, demonstrating that use of our algorithm on large and complicated systems is quite feasible.

Berry curvature from ramps - Consider an arbitrary manifold of Hamiltonians, $H(\vec{\lambda})$, parameterized by some externally-controlled parameters $\vec{\lambda}$ (e.g., magnetic field). Given the ground states $\left|\psi_{0}(\vec{\lambda})\right\rangle$, the Berry phase is defined for some closed loop $\mathcal{C}$ in parameter space as follows. Consider ramping the parameters around this loop adiabatically and returning to the initial point $\vec{\lambda}_{i}$. A naive expectation is that the wave function will return to $\left|\psi_{0}\left(\vec{\lambda}_{i}\right)\right\rangle$ up to a dynamical phase factor $e^{i \gamma_{\mathrm{dyn}}}$, where $\gamma_{\mathrm{dyn}}=-\int E_{0} d t$. However, there is an additional phase factor $\gamma_{B}$ known as the geometric or Berry phase [1, which derives from the quantum geometry of the ground state manifold. The Berry phase is given by

$$
\gamma_{B}=i \oint_{\mathcal{C}}\left\langle\psi_{0}|\vec{\nabla}| \psi_{0}\right\rangle \cdot d \vec{\lambda} \equiv \oint_{\mathcal{C}} \vec{A} \cdot d \vec{\lambda}
$$

where $\vec{A}$ is the Berry connection. If we think of $\vec{A}$ as a magnetic vector potential, then its curl is the "magnetic field" $F$, called the Berry curvature:

$$
F_{\mu \nu}=\partial_{\mu} A_{\nu}-\partial_{\nu} A_{\mu} .
$$

Just as the phase of a charged particle acquires an 
Aharonov-Bohm phase when moving around a magnetic flux, the surface integral of the Berry curvature over a manifold $\mathcal{M}$ with $\mathcal{C}$ as its boundary gives the Berry phase: $\gamma_{B}=\int_{\mathcal{M}} F \cdot d S$. If $\mathcal{M}$ is a closed manifold, then singlevaluedness of the wave function demands that the phase be $2 \pi n$ for some integer

$$
n=\frac{1}{2 \pi} \oint_{\mathcal{M}} F \cdot d S
$$

where $n$ is a topological invariant known as the first Chern number. In the language of effective magnetic field, $n$ is the number of flux quanta piercing the surface $\mathcal{M}$, which is an integer due to Dirac monopole quantization 36. It can be used to define the topological order of many materials, e.g., quantum Hall systems and topological insulators [4, 9, 11, 13. Therefore, the Berry curvature plays a crucial role in the geometry and topology of the ground state manifold.

Recently, Gritsev and Polkovnikov 35] pointed out that the Berry curvature emerges naturally as a leading order corrections to adiabatic dynamics in ramped systems. While Monte Carlo methods are unable to simulate such real-time dynamics, a similar analysis in imaginary time 26] gives the Berry curvature for asymmetric ramps. More explicitly, consider an imaginary time ramp along some direction $\lambda_{\mu}$ at rate $v_{\mu}=d \lambda_{\mu} / d t$. Then, stopping at a fixed point $\vec{\lambda}_{f}$ in parameter space, the wave function to lowest order in $v_{\mu}$ is given by 37

$$
\left|\psi\left(v_{\mu}\right)\right\rangle \approx|0\rangle-v_{\mu} \sum_{n \neq 0}|n\rangle \frac{\left\langle n\left|\partial_{\mu} H\right| 0\right\rangle}{\left(E_{n}-E_{0}\right)^{2}}+O\left(v_{\mu}^{2}\right)
$$

where $|n\rangle$ labels the energy eigenstates of $H\left(\vec{\lambda}_{f}\right)$ with energy $E_{n}$ and non-degenerate ground state $|0\rangle$. Now imagine propagating the bra and ket asymmetrically, and taking the matrix element of the generalized force $\partial_{\nu} H$. Noting that $\left\langle\psi\left(-v_{\mu}\right) \mid \psi\left(v_{\mu}\right)\right\rangle=1$ to order $v_{\mu}^{2}$, we find the leading contribution to this overlap is

$$
\frac{\left\langle\psi\left(-v_{\mu}\right)\left|\partial_{\nu} H\right| \psi\left(v_{\mu}\right)\right\rangle}{\left\langle\psi\left(-v_{\mu}\right) \mid \psi\left(v_{\mu}\right)\right\rangle} \approx\left\langle 0\left|\partial_{\nu} H\right| 0\right\rangle-i v_{\mu} F_{\mu \nu}+O\left(v_{\mu}^{2}\right)
$$

where $F_{\mu \nu}$ is the (many-body) Berry curvature 50. Since $\partial_{\nu} H$ is Hermitian, the first term in Eq. 5 is real, while the second term is strictly imaginary. Thus,

$$
\begin{aligned}
v_{\mu} F_{\mu \nu} & \approx-\operatorname{Im}\left[\frac{\left\langle\psi\left(-v_{\mu}\right)\left|\partial_{\nu} H\right| \psi\left(v_{\mu}\right)\right\rangle}{\left\langle\psi\left(-v_{\mu}\right) \mid \psi\left(v_{\mu}\right)\right\rangle}\right] \\
& =\operatorname{Re}\left[\frac{\left\langle\psi\left(-v_{\mu}\right)\left|\left(i \partial_{\nu} H\right)\right| \psi\left(v_{\mu}\right)\right\rangle}{\left\langle\psi\left(-v_{\mu}\right) \mid \psi\left(v_{\mu}\right)\right\rangle}\right] .
\end{aligned}
$$

By taking the slow limit of these asymmetric ramps and measuring the generalized force $i \partial_{\nu} H$, we can extract the Berry curvature. These imaginary time ramps are amenable to QMC methods, as long as the Hamiltonian is sign free for all values of $\lambda_{\mu}$ during the ramp. As a demonstration of this method, we now construct an algorithm for computing Eq. 6] in the TFI model using an extension of the quasi-adiabatic QMC method [26].

Application to the TFI model - Consider the TFI model on a $d$-dimensional lattice, with Hamiltonian

$$
H=-J \sum_{<j j^{\prime}>} \sigma_{j}^{x} \sigma_{j^{\prime}}^{x}-h \sum_{j} \sigma_{j}^{z}
$$

where $J>0$ is the ferromagnetic Ising interaction acting on nearest neighbors $j$ and $j^{\prime}, h$ is the transverse field, and $\sigma^{x, y, z}$ are Pauli matrices. The ground state of this Hamiltonian has a quantum phase transition from paramagnet to ferromagnet at the critical point $h_{c}$, where $h_{c}= \pm J$ in one dimension [22] and $h_{c} \approx \pm 3.04458 J$ in two dimensions [26]. To get non-zero Berry curvature, we introduce a third parameter $\phi$ corresponding to a global rotation of all the spins by an angle $\phi / 2$ about the $z$-axis. The Berry phase of this extended TFI model has been investigated experimentally 38, and theoretically [39, 40] in the integrable one-dimensional case. Here, we numerically extend this analysis to arbitrary dimensionality $d$ by using QMC methods.

To fix the overall energy scale, we reparameterize the couplings as $h=s$ and $J=1-s$. Then the Hamiltonian described above can be written

$H(s, \phi)=-s \sum_{j} \sigma_{j}^{x}-(1-s) \sum_{<j j^{\prime}>}\left[\sigma_{j}^{+} \sigma_{j^{\prime}}^{-}+e^{i \phi} \sigma_{j}^{+} \sigma_{j^{\prime}}^{+}+\right.$h.c. $]$

where $\sigma^{ \pm}=\frac{1}{2}\left(\sigma^{z} \pm i \sigma^{y}\right)$. We are interested in the non-trivial component $F_{s \phi}$ of the Berry curvature tensor, which is a function of the tuning parameter $s$. For this choice of parameters, consider a ramp from $s=0$ to $s=1$. At $s=1$, the ground state consists of all spins pointing to the right, which we denote $|\Rightarrow\rangle$. Similarly, at $s=0$, the ground state consists of either all states pointing up or all down; we manually break the symmetry by choosing $|\Uparrow\rangle$ [51]. Then we wish to use QMC to measure the overlap in Eq. 6] as a function of $s \in(0,1)$ : 


$$
\left\langle i \partial_{\phi} H\right\rangle_{\text {asym }} \equiv \frac{\left\langle\psi(-v)\left|\left(i \partial_{\phi} H\right)\right| \psi(v)\right\rangle}{\langle\psi(-v) \mid \psi(v)\rangle} \approx \frac{\left\langle\Rightarrow\left|e^{-H_{M} \delta \tau} e^{-H_{M-1} \delta \tau} \cdots e^{-H_{m} \delta \tau}\left[i \partial_{\phi} H\right] e^{-H_{m-1} \delta \tau} \cdots e^{-H_{1} \delta \tau}\right| \Uparrow\right\rangle}{\left\langle\Rightarrow\left|e^{-H_{M} \delta \tau} e^{-H_{M-1} \delta \tau} \cdots e^{-H_{m} \delta \tau}[\mathbb{1}] e^{-H_{m-1} \delta \tau} \cdots e^{-H_{1} \delta \tau}\right| \Uparrow\right\rangle} \approx v F_{s \phi},
$$

where we have discretized the imaginary time evolution using an $M$-step Trotter decomposition with time step $\delta \tau$. The velocity is $v=\delta s / \delta \tau=1 /(M \delta \tau)$, while the Hamiltonians at each step are $H_{p} \equiv H\left(s=\frac{p-1 / 2}{M}\right)$. To implement this ramp more easily, we use a trick from quasi-adiabatic QMC (QAQMC) and approximate Eq. 9 by [26]

$$
\left\langle i \partial_{\phi} H\right\rangle_{\text {asym }} \approx \frac{\left\langle\Rightarrow\left|\left(-H_{M}\right)\left(-H_{M-1}\right) \cdots\left(-H_{m}\right)\left[i \partial_{\phi} H\right]\left(-H_{m-1}\right) \cdots\left(-H_{1}\right)\right| \Uparrow\right\rangle}{\left\langle\Rightarrow\left|\left(-H_{M}\right)\left(-H_{M-1}\right) \cdots\left(-H_{m}\right)[\mathbb{1}]\left(-H_{m-1}\right) \cdots\left(-H_{1}\right)\right| \Uparrow\right\rangle},
$$

where the effective ramp rate is now $v \approx-E_{0} / M$, with $E_{0}$ the ground state energy of $H$ at the measurement point $s_{\text {meas }}=m / M$. This expression becomes exact in the limit of $M, E_{0} \rightarrow \infty$, which can be achieved by taking the limit of large system size $L$.

In order to use an SSE-like method to extract $F_{s \phi}$, we split the Hamiltonian up into bond and site operators (after adding a constant offset):

$H(s, \phi=0)=-s \sum_{j}\left[\mathbb{1}_{j}-\sigma_{j}^{x}\right]-(1-s) \sum_{<j j^{\prime}>}\left[\sigma_{j}^{z} \sigma_{j^{\prime}}^{z}+\mathbb{1}_{j j^{\prime}}\right]$,

as in equilibrium SSE-QMC simulations of the Ising model [4]. We also must introduce operators corresponding to the generalized force,

$$
\begin{aligned}
\left.\partial_{\phi} H\right|_{\phi=0} & =i(1-s) \sum_{<j j^{\prime}>}\left(\sigma_{j}^{+} \sigma_{j^{\prime}}^{+}-\sigma_{j}^{-} \sigma_{j^{\prime}}^{-}\right) \\
& =-\frac{1-s}{2} \sum_{<j j^{\prime}>}\left[\sigma_{j}^{y} \sigma_{j^{\prime}}^{z}+\sigma_{j}^{z} \sigma_{j^{\prime}}^{y}\right] .
\end{aligned}
$$

To this end, we define the measurement operator

$$
\begin{aligned}
\mathcal{M} & =\sum_{<j j^{\prime}>}\left[\mathbb{1}_{j j^{\prime}}-i \sigma_{j}^{y} \sigma_{j^{\prime}}^{z}-i \sigma_{j}^{z} \sigma_{j^{\prime}}^{y}+\sigma_{j}^{x} \sigma_{j^{\prime}}^{x}\right] \\
& =N_{\text {bond }} \mathbb{1}+\frac{2}{1-s}\left(i \partial_{\phi} H\right)+H_{x x} .
\end{aligned}
$$

Here the identity term $\mathbb{1}$ is used in sampling the denominator of Eq. 10, the generalized force term $i \partial_{\phi} H$ is used for the numerator, and the spurious $H_{x x}$ term is included to maximize ergodicity. Finally, we measure the ground state energy $E_{0}$ within the same simulation by sampling

$$
\frac{\left\langle\Rightarrow\left|\left(-H_{M}\right) \cdots\left[H_{m}\right] \cdots\left(-H_{1}\right)\right| \Uparrow\right\rangle}{\langle\Rightarrow|\cdots[\mathbb{1}] \cdots| \Uparrow\rangle}=E_{0}+O\left(v^{2}\right) .
$$

We efficiently sample the overlaps in Eqs. 10 and 14 via cluster updates similar to those in conventional SSEQMC [41; details of the algorithm, including the cluster updates, can be found in the appendix. In addition to standard diagonal and cluster updates for the Ising

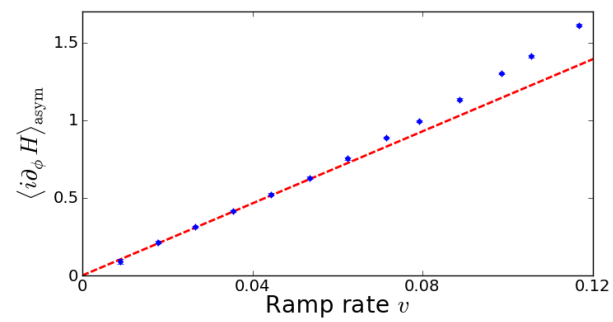

FIG. 1: To extract the Berry curvature of the one dimensional TFI model from QMC, we plot the generalized force $\left\langle i \partial_{\phi} H\right\rangle_{\text {asym }}$ as a function of ramp rate $v_{\text {asym }}$ for $s_{\text {meas }}=0.25$ and $L=50$. The slope at low velocity gives the Berry curvature $F_{s \phi}$, which matches well with the exact result (dashed line).

Hamiltonians, we introduce additional updates to sample the operators from $\mathcal{M}$. The crucial idea there is that the $\sigma^{y} \sigma^{z}$ terms in $i \partial_{\phi} H$ are "half-diagonal," i.e. $\sigma^{y}$ flips the spin and $\sigma^{z}$ does not. Therefore, for the cluster update, we treat the operators in $\mathcal{M}$ as two separate sites, which are updated according to the same rules as the onsite $\sigma^{x}$ and $\mathbb{1}$ operators. So, for example, if the incoming vertex is to the $\sigma_{j^{\prime}}^{z}$ vertex of a $\sigma_{j}^{y} \sigma_{j^{\prime}}^{z}$ operator, then the vertex is flipped to give $\sigma_{j}^{x} \sigma_{j^{\prime}}^{x}$ [52].

The simple method as described above works well for many cases, but devotes unnecessary time to ramping through values of $s$ far from the measurement point. We therefore improve the algorithm by starting with $s_{i}$ and $s_{f}$ closer $s_{\text {meas }}$, zooming in on a range of small width $\Delta s=s_{f}-s_{i}$ around it [53]. By changing $s_{i}$ and $s_{f},\langle\Rightarrow|$ and $|\Uparrow\rangle$ are no longer the ground states at the boundaries. In principle, one could modify the algorithm to first project to the ground state at each end; however, as we are performing imaginary time ramps, the dynamics will continually project toward the ground state. Therefore, the boundary states don't matter for slow enough ramps [37, and our algorithm works as before if $M$ is large enough to allow the initial transients to relax [54].

For a given $s_{\text {meas }}$, as the operators $\left(-H_{p}\right)$ are applied $M$ times, physical observables relax to their ground state value roughly exponentially [42] with decay rate propor- 


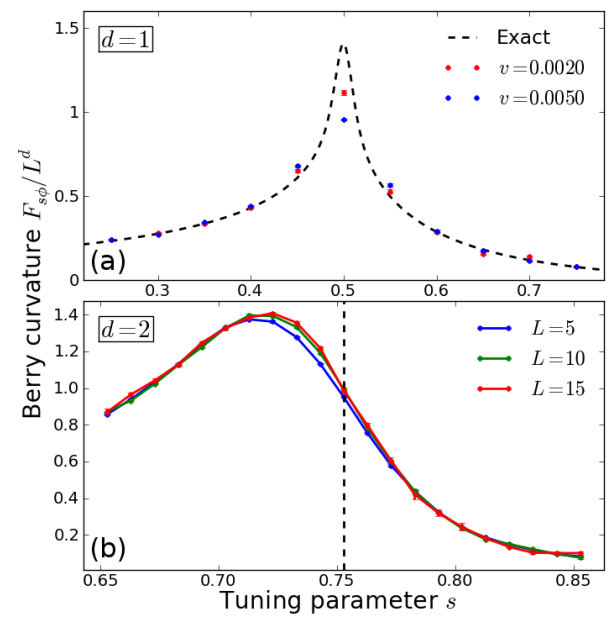

FIG. 2: Berry curvature of the TFI model in one (a) and two (b) dimensions. (a) Results for one-dimensional model on an $L=50$ site lattice at two small ramp rates. The dashed line shows the exact result $(v \rightarrow 0)$ for comparison. (b) Measured Berry curvature of the $d=2$ TFI model as a function of system size at fixed $v \sim L^{d} \Delta s / M=10^{-2}$. Unlike the onedimensional case, the Berry curvature does not diverge in the thermodynamic limit.

tional to the (many-body) energy gap. Therefore, to approach the limit $M \rightarrow \infty$ where the algorithm becomes exact, we measure $F_{s \phi}$ while varying $M$, and perform an exponential fit to estimate the decay constant $M_{\text {decay }}$, and subsequently work with $M$ much larger than $M_{\text {decay }}[55]$.

With this improvement, we now see that our QMC method scales similarly to conventional ground state methods. As noted earlier, the number of steps required to reach the ground state scales as $M \sim L^{d} / \Delta$ [42]. Then, to obtain a given velocity, we simply tune the range $\Delta s$. Therefore, the scaling of the number of steps $M$ to get a given velocity is $M \sim \Delta^{-1}$, as opposed to $M \sim \Delta^{-2}$ as might be expected from more naive methods (to get $v \ll \Delta^{2}$ ). In addition, there is only one step (the measurement) which involves signed sums, and this "sign problem" does not scale with system size. Therefore, there is no exponentially bad sign problem; indeed, since the scaling of $M$ is dominated by the number of steps required to reach the ground state, it is identical to similar ground state algorithms.

Results - For the one-dimensional case, the TFI model is exactly solvable via Jordan-Wigner transformation [22, from which the Berry curvature can easily be obtained numerically [40]. As an initial test of the algorithm, we check that the exact and QMC solutions match for an example point in Fig. 1. As expected, the generalized force $i \partial_{\phi} H$ scales linearly with $v$ at low ramp rates, with slope given by the Berry curvature.

We now proceed to obtain the Berry curvature of the full phase diagram in the one-dimensional model with
$L=50$ and in two dimensions for a range of $L$ (Fig. 2). The $1 \mathrm{D}$ results are compared to the exact solution, where clear deviations are seen at finite velocity. This is not surprising, given that the adiabatic limit requires that the velocity be less than the gap squared: $v \ll \Delta^{2}[43$. So, given that the gap is vanishes at the critical point $(s=1 / 2)$ in the thermodynamic limit, the finite-velocity deviations are strongest near there [56. However, for the case of a gapped spectrum, low velocities are already sufficient to get the Berry curvature.

Having established that the method works in the integrable one-dimensional case, we can now easily do the calculation for the non-integrable case of two dimensions. The results of this simulation are shown in Fig. 2p. We immediately see a qualitative difference from the onedimensional model: the Berry curvature no longer is divergent (in the thermodynamic limit) at the quantum critical point. Indeed, unlike in one dimension, the twodimensional Berry curvature appears to approach the thermodynamic limit for systems as small as $L=10$.

We can understand this difference using critical scaling arguments. As shown in Ref. 44, the scaling dimension of the Berry curvature is given by $F_{s \phi} / L^{d} \sim \mid s-$ $\left.s_{c}\right|^{-\nu_{s}\left(d+2 z-\Delta_{s}-\Delta_{\phi}\right)} \equiv\left|s-s_{c}\right|^{\alpha}$, where $\Delta_{\lambda}=d+z-1 / \nu_{\lambda}$ is the scaling of the operator $\partial_{\lambda} H$. For the one-dimensional TFI model, we get $\alpha=0$ (i.e., $F_{s \phi} / L \sim \log \left(\left|s-s_{c}\right|\right)$ ), while for two dimensions (using the exponents in Ref.26), we get $\alpha \approx 0.258$, which is singular but not divergent. Thus, the 2D Berry curvature will be some smooth function of $s$ plus a non-divergent singularity at the critical point. While our simulations are unable to resolve this singularity, Fig. 2 $\mathrm{b}$ is consistent with its existence and clearly rules out the existence of a divergent Berry curvature at the critical point.

Conclusions - In conclusion, we have implemented a QMC method to measure Berry curvature. Despite the fact that Berry curvature measures properties of the ground state Berry phase, our algorithm has no sign problem for the same set of Hamiltonians as more conventional QMC methods. In addition, the algorithm scales efficiently with both system size and energy gap. Using our specific implementation similar to quasi-adiabatic QMC, we solved for the ground state Berry curvature of the transverse-field Ising model in one and two dimensions. In agreement with critical scaling theory, we saw a qualitative distinction between the models, namely the presence (absence) of divergence in the Berry curvature in one (two) dimensions.

To our knowledge, this is the first demonstration of a QMC method to solve for the Berry curvature with respect to global (as opposed to local [45]) coupling parameters. Having seen that this idea works for a simple case, the idea is readily extensible to other models. Possible extensions include understanding the response to twisted boundary conditions ("flux insertion" 46]) and topological phase transitions in bosons [20, spins [27, or even 
fermions 34. An interesting open question is whether a small ramp along a direction with a sign problem is sufficient to create a sign problem in the algorithm. If it is possible to do small ramps around a sign-problemfree point, this would further open the class of problems solvable via such a method.

Acknowledgments - We thank Anatoli Polkovnikov and Anders Sandvik for crucial discussions and ChengWei Liu for sharing his QAQMC code. We gratefully acknowledge financial support from AFOSR grant number FA9550-10-1-0110 and NSF grant number PHY-1211284.

[1] M. V. Berry, Proc. Roy. Soc. A 392, 45 (1984).

[2] Y. Aharonov and J. Anandan, Phys. Rev. Lett. 58, 1593 (1987).

[3] F. D. M. Haldane, Phys. Rev. Lett. 61, 2015 (1988).

[4] C. L. Kane and E. J. Mele, Phys. Rev. Lett. 95, 146802 (2005).

[5] B. A. Bernevig, T. L. Hughes, and S.-C. Zhang, Science 314, 1757 (2006).

[6] M. Knig, S. Wiedmann, C. Brne, A. Roth, H. Buhmann, L. W. Molenkamp, X.-L. Qi, and S.-C. Zhang, Science 318, 766 (2007).

[7] L. Fu, C. L. Kane, and E. J. Mele, Phys. Rev. Lett. 98, 106803 (2007).

[8] D. Hsieh, D. Qian, L. Wray, Y. Xia, Y. S. Hor, R. J. Cava, and M. Z. Hasan, Nature 452, 970 (2008), ISSN 00280836, URL http://dx.doi.org/10.1038/nature06843.

[9] D. J. Thouless, M. Kohmoto, M. P. Nightingale, and M. den Nijs, Phys. Rev. Lett. 49, 405 (1982).

[10] Q. Niu, D. J. Thouless, and Y.-S. Wu, Phys. Rev. B 31, 3372 (1985).

[11] Y. Hatsugai, Phys. Rev. Lett. 71, 3697 (1993).

[12] C. M. Canali, A. Cehovin, and A. H. MacDonald, Phys. Rev. Lett. 91, 046805 (2003).

[13] D. N. Sheng, Z. Y. Weng, L. Sheng, and F. D. M. Haldane, Phys. Rev. Lett. 97, 036808 (2006).

[14] M. Levin and X.-G. Wen, Rev. Mod. Phys. 77, 871 (2005).

[15] F. D. M. Haldane, Phys. Rev. Lett. 93, 206602 (2004).

[16] R. D. King-Smith and D. Vanderbilt, Phys. Rev. B 47, 1651 (1993).

[17] R. Resta, Journal of Physics: Condensed Matter 12, R107 (2000), ISSN 0953-8984.

[18] M. Hafezi, A. S. Srensen, M. D. Lukin, and E. Demler, EPL (Europhysics Letters) 81, 10005 (2008), ISSN 02955075 .

[19] B. L. Hammond, W. A. J. Lester, and P. J. Reynolds, Monte Carlo Methods In Ab Initio Quantum Chemistry (World Scientific, 1994).

[20] M. Nightingale and C. J. Umrigar, eds., Quantum Monte Carlo Methods in Physics and Chemistry (Springer, 1999).

[21] J. B. Anderson, Quantum Monte Carlo: Origins, Development, Applications (Oxford University Press, 2007).

[22] E. Lieb, T. Schultz, and D. Mattis, Annals of Physics 16, 407 (1961), ISSN 0003-4916.

[23] P. Pfeuty and R. J. Elliott, Journal of Physics C: Solid State Physics 4, 2370 (1971), ISSN 0022-3719.
[24] M. S. L. du Croo de Jongh and J. M. J. van Leeuwen, Phys. Rev. B 57, 8494 (1998).

[25] C. J. Hamer, Journal of Physics A: Mathematical and General 33, 6683 (2000), ISSN 0305-4470.

[26] C.-W. Liu, A. Polkovnikov, and A. W. Sandvik, Phys. Rev. B 87, 174302 (2013).

[27] O. F. Syljuasen and A. W. Sandvik, Phys. Rev. E 66, 046701 (2002).

[28] A. W. Sandvik, Phys. Rev. Lett. 98, 227202 (2007).

[29] R. G. Melko and R. K. Kaul, Phys. Rev. Lett. 100, 017203 (2008).

[30] D. M. Ceperley, Rev. Mod. Phys. 67, 279 (1995).

[31] B. K. Clark and D. M. Ceperley, Phys. Rev. Lett. 96, 105302 (2006).

[32] M. Boninsegni and N. V. Prokofev, Rev. Mod. Phys. 84, 759 (2012).

[33] G. Sugiyama and S. Koonin, Annals of Physics 168, 1 (1986), ISSN 0003-4916.

[34] S. Sorella, S. Baroni, R. Car, and M. Parrinello, EPL (Europhysics Letters) 8, 663 (1989), ISSN 0295-5075.

[35] V. Gritsev and A. Polkovnikov, Proceedings of the National Academy of Sciences 109, 6457 (2012).

[36] P. Dirac, Proc. Roy. Soc. A 133, 60 (1931).

[37] C. De Grandi, A. Polkovnikov, and A. W. Sandvik, Phys. Rev. B 84, 224303 (2011).

[38] X. Peng, S. Wu, J. Li, D. Suter, and J. Du, Phys. Rev. Lett. 105, 240405 (2010).

[39] S.-L. Zhu, Phys. Rev. Lett. 96, 077206 (2006).

[40] M. Kolodrubetz, V. Gritsev, and A. Polkovnikov, Phys. Rev. B 88, 064304 (2013).

[41] A. W. Sandvik, Phys. Rev. E 68, 056701 (2003).

[42] A. W. Sandvik and H. G. Evertz, Phys. Rev. B 82, 024407 (2010).

[43] C. De Grandi and A. Polkovnikov, Quantum Quenching, Annealing and Computation, vol. 802 (Springer, 2010).

[44] L. Campos Venuti and P. Zanardi, Phys. Rev. Lett. 99, 095701 (2007).

[45] Y. Motoyama and S. Todo, Phys. Rev. E 87, 021301 (2013).

[46] F. F. Assaad, W. Hanke, and D. J. Scalapino, Phys. Rev. Lett. 71, 1915 (1993).

[47] A. Shapere and F. Wilczek, Geometric Phases In Physics (World Scientific, 1989).

[48] P. Zanardi and M. Rasetti, Physics Letters A 264, 94 (1999), ISSN 0375-9601.

[49] J. Pachos, P. Zanardi, and M. Rasetti, Phys. Rev. A 61, 010305 (1999).

[50] To show this, we use the form of the Berry curvature in terms of generalized forces: $F_{\mu \nu}=$ $i \sum_{n \neq 0} \frac{\left\langle 0\left|\partial_{\mu} H\right| n\right\rangle\left\langle n\left|\partial_{\nu} H\right| 0\right\rangle-(\mu \leftrightarrow \nu)}{\left(E_{n}-E_{0}\right)^{2}}[1]$.

[51] Note that, by breaking the $\mathbb{Z}_{2}$ symmetry, we expect to measure the Berry curvature with respect to the ground state manifold with the chosen magnetization (in the limit of large system size, i.e., exponentially small gap between ground state sectors). This is important because formally each element of the Berry curvature tensor is itself an $n \times n$ matrix known as the non-Abelian Berry curvature in the case of $n$ degenerate ground states 47 49. However, we use the fact that the Berry curvature should not depend on the choice of ground state sector to explicitly solve for only the case of positive magnetization along the $z$ direction. We note that similar choices are employed in other analytic studies of the ground state 
geometry of TFI chain 39, 40.

[52] For the purposes of QMC sampling, $\sigma^{x}$ and $\sigma^{y}$ are treated the same, since they both flip the spin with the same absolute values of their matrix elements. The difference enters in the sign of the measurement; see appendix for details.

[53] For this paper, we specifically choose the symmetric case $s_{i}=s_{\text {meas }}-\Delta s / 2$ and $s_{f}=s_{\text {meas }}+\Delta s / 2$

[54] Indeed, with this modification the identity of the boundary states no longer matters at all, and one could choose them as desired for numerical convenience. For the remainder of this paper, we stick with the original boundary conditions $|\Rightarrow\rangle$ and $|\Uparrow\rangle$ for convenience.

[55] We use roughly $M \approx 10 M_{\text {decay. }}$.

[56] When working with finite values of the velocity and system size, we can extend this analysis by performing Kibble-Zurek scaling of the Berry curvature to near the critical point 26].

\section{Appendix: Details of QMC scheme}

In this section, we detail the QMC scheme used to solve for the Berry curvature of the TFI model. With the exception of the measurement operator $\mathcal{M}$, it is very similar to the algorithm found in Ref. 26, which in turn is based on SSE-QMC for the TFI model [41]. We start by briefly reviewing the QMC update steps for the Hamiltonians, then discuss how this is modified by the presence of $\mathcal{M}$. Finally, we discuss the computation of observables, namely the generalized force $i \partial_{\phi} H$ and the ground state energy $E_{0}$.

In the SSE method, the Monte Carlo configuration consists of an operator string with one operator for each step $p$. Throughout this appendix, we discuss the improved version of the algorithm, in which $s_{i}=s_{\text {meas }}-\frac{\Delta s}{2}$ and $s_{f}=s_{\text {meas }}+\frac{\Delta s}{2}$. Therefore, step $p$ samples from the Hamiltonian $H_{p} \equiv H\left(s=s_{p}\right)$, where $s_{p}=s_{i}+$ $\left(\frac{p-1 / 2}{M}\right) \Delta s$. We denote by $H_{i_{p}}^{p}$ such an operator sampled from $H_{p}$, where $i_{p}$ iterates over the possible operators in Eq. 11. SSE generally requires sampling over spin states at the boundaries, but as we'll see, this is unnecessary in the current case. We sample an additional operator for the measurement, which we denote $\mathcal{M}_{i_{\mathcal{M}}}$. So for our algorithm, a configuration $c$ is represented by the operator string $\left(H_{i_{1}}^{1}, \ldots, H_{i_{M}}^{M}, \mathcal{M}_{i_{\mathcal{M}}}\right)$.

Given the desired measurement (Eq. 10), we define the sampling weight $w$ for configuration $c$ to be proportional to

$$
w(c) \propto\left\langle\Rightarrow\left|\left(-H_{i_{1}}^{1}\right) \cdots\left(-H_{i_{m}}^{m}\right)\left(\mathcal{M}_{i_{\mathcal{M}}}\right) \cdots\left(-H_{i_{M}}^{M}\right)\right| \Uparrow\right\rangle .
$$

These weights can be negative because the measurement operator has terms of the form $i \sigma^{y} \sigma^{z}$ which have negative matrix elements. Therefore, the sampling probability is just the amplitude of this weight: $p(c)=|w(c)|$.

Configurations can be efficiently sampled according to this probability distribution using a straightforward ex- tension of SSE cluster updates. The basic QMC step of SSE-QMC on the TFI model consists of two parts: the diagonal update, followed by the off-diagonal cluster update 41. The diagonal update only re-samples operators that are diagonal in the spin basis. Since we choose to quantize the spins along the $z$ axis, the diagonal operators in Eq. 11 are the Ising operator and the heat bath operator $\mathbb{1}_{j}$. At each step $p$ for which one of these operators is in the operator string (i.e., the current Monte Carlo configuration), a new diagonal operator is selected at random such that the new configuration $c^{\prime}$ is selected with probability proportional to $p\left(c^{\prime}\right)$. The heat bath positions are therefore selected at random, while the Ising bond is only allowed to be inserted in positions where the spins on either side of the bond are aligned. The diagonal update of $\mathcal{M}$ is also straightforward, since the only diagonal operator is $\mathbb{1}_{j j^{\prime}}$. Therefore, if the operator $\mathcal{M}_{i \mathcal{M}}$ starts as an identity, we move it to a random bond during the diagonal update step.

The cluster update is more complicated, and is responsible for the efficiency of the SSE algorithm. The idea is to generate a connected cluster of "nodes," where each node is an entrance or an exit vertex from a given operator. Then, with probability $p=1 / 2$, each cluster is flipped, meaning that the spin state at each node is flipped with the operator changed accordingly. The rules for generating the cluster are simple: nodes that enter a heat bath $\left(\mathbb{1}_{j}\right)$ or spin flip $\left(\sigma_{j}^{z}\right)$ operator terminate the cluster, while nodes entering an Ising bond continue the cluster growth from the other three nodes on that Ising operator [41. The $50 \%$ acceptance rate comes from the fact that a cluster has the same sampling probability $p(c)$ before and after flipping. There is one slight difference between our situation and that of some other algorithms: one of our boundaries has all spins pointing up $(|\Uparrow\rangle)$, meaning that clusters that are in contact with that boundary are not flipped. This is in contrast to the other boundary $|\Rightarrow\rangle$, where clusters touching the boundary can be flipped because the $|\Rightarrow\rangle$ state has equal overlap with all spin states in $\uparrow / \downarrow$ basis.

In extending these ideas to the measurement step, given by the operator $\mathcal{M}$ in Eq. 13 , the tricky part is that the $\sigma^{y} \sigma^{z}$ terms are "half-diagonal." They are therefore similar in spirit to two copies of the site operators $\sigma_{j}^{x}$ and $\mathbb{1}_{j}$. By introducing the otherwise useless $\sigma^{x} \sigma^{x}$ operator, which flips both sites, we can complete this analogy by doing the cluster update of all terms in $\mathcal{M}$ by just treating them as two separate site operators, which just happen to lie on the same bond. For example, if we start with operator $\sigma_{j}^{y} \sigma_{j^{\prime}}^{z}$ on some bond $\left\langle j j^{\prime}>\right.$ and generate a cluster that comes in with entrance vertex on site $j^{\prime}$, then we stop the cluster upon entering the $\sigma^{y} \sigma^{z}$ vertex. If the cluster is subsequently chosen to be flipped, we flip the operator at site $j^{\prime}$ to get $\sigma_{j}^{x} \sigma_{j^{\prime}}^{x}$. As before, the clusters can be flipped with probability $1 / 2$, because the amplitude $p(c)$ is the same before and after flipping. Note 
that, in flipping the operators, $\sigma^{y}$ and $\sigma^{x}$ are treated as identical because they have the same magnitude of their matrix elements (i.e., they give the same $p(c)$ ). The signs that distinguish these Pauli matrices enter into the actual measurement.

In taking operator expectation values (with respect to $p(c)$ ), the sign of the weight $w(c)$ must be considered. More explicitly, the asymmetric expectation value of $i \partial_{\phi} H$ can be measured using the indicator observables

$$
\begin{aligned}
& \mathcal{O}_{y z}(c)= \begin{cases}\operatorname{sgn}[w(c)] & \text { if } \mathcal{M}_{i_{\mathcal{M}}} \in\left\{\sigma_{j}^{z} \sigma_{j^{\prime}}^{y}\right\} \\
0 & \text { otherwise }\end{cases} \\
& \mathcal{O}_{\mathbb{1}}(c)= \begin{cases}1 & \text { if } \mathcal{M}_{i_{\mathcal{M}}} \in\left\{\mathbb{1}_{j j^{\prime}}\right\} \\
0 & \text { otherwise }\end{cases}
\end{aligned}
$$

Then the overlap is given by

$$
\left\langle i \partial_{\phi} H\right\rangle_{\text {asym }}=\frac{N_{\text {bond }}(1-s)}{2} \frac{\left\langle\mathcal{O}_{y z}\right\rangle_{p(c)}}{\left\langle\mathcal{O}_{\mathbb{1}}\right\rangle_{p(c)}},
$$

where $\langle\cdots\rangle_{p(c)}$ is the statistical expectation value and the $\frac{N_{\text {bond }}(1-s)}{2}$ term comes from the prefactor in Eq. 13

To obtain the velocity $v \approx-E_{0} / M$, we need access to the ground state energy $E_{0}$. Rather than separately solving this energy, we use the approximate form

$$
E(v) \equiv \frac{\langle\psi(-v)|H| \psi(v)\rangle}{\langle\psi(-v)| \psi(v)},
$$

which can be obtained at the same time as we measure the asymmetric expectation value $\left\langle i \partial_{\phi} H\right\rangle_{\text {asym }}$. Furthermore, since this observable is diagonal in the energy basis, the leading order correction will be of order $v^{2}$ [37], so that $E(v)=E_{0}+O\left(v^{2}\right)$. Therefore, in the limit $v \rightarrow 0$, using $E(v)$ in place of $E_{0}$ in the formula for $F_{s \phi}$ should still be accurate to order $v$.

The energy consists of two terms: an Ising energy that is diagonal in the $z$ basis and a field energy that is offdiagonal. Diagonal and off-diagonal operators are generally measured differently in SSE-QMC [27. In particular, while both energies are measured only for the case where $\mathcal{M}_{i_{M}}$ is an identity matrix, the Ising energy is measured precisely at the measurement step $m$, while the field energy is averaged over steps $m-1$ and $m$. More explicitly, the indicator observable for the Ising energy is

$$
\mathcal{O}_{\text {ising }}(c)=\left\{\begin{array}{ll}
\sum_{<j j^{\prime}>} \eta_{j j^{\prime}} & \text { if } \mathcal{M}_{i \mathcal{M}} \in\left\{\mathbb{1}_{j j^{\prime}}\right\} \\
0 & \text { otherwise }
\end{array},\right.
$$

where $\eta_{j j^{\prime}}=1$ if the spin states on sites $j$ and $j^{\prime}$ match in the configuration $c$ and $\eta_{j j^{\prime}}=-1$ otherwise. Similarly, the field energy at step $p$ is given by the indicator variables

$$
\begin{aligned}
& \mathcal{O}_{\text {field }}^{p}(c)= \begin{cases}1 & \text { if } \mathcal{M}_{i_{\mathcal{M}}} \in\left\{\mathbb{1}_{j j^{\prime}}\right\} \text { and } H_{i_{p}}^{p} \in\left\{\sigma_{j}^{x}\right\}_{(21)} \\
0 & \text { otherwise }\end{cases} \\
& \mathcal{O}_{\text {bath }}^{p}(c)= \begin{cases}1 & \text { if } \mathcal{M}_{i_{\mathcal{M}}} \in\left\{\mathbb{1}_{j j^{\prime}}\right\} \text { and } H_{i_{p}}^{p} \in\left\{\mathbb{1}_{j}\right\} \\
0 & \text { otherwise }\end{cases}
\end{aligned}
$$

Then the Ising and field energy densities at the point $s_{\text {meas }}$ are

$$
\frac{E_{\text {ising }}}{N_{\text {site }}}=\frac{J_{z}\left\langle\mathcal{O}_{\text {ising }}\right\rangle_{p(c)}}{N_{\text {bond }}\left\langle\mathcal{O}_{\mathbb{1}}\right\rangle_{p(c)}} \quad, \quad \frac{E_{\text {field }}}{N_{\text {site }}}=\frac{h\left\langle\frac{\mathcal{O}_{\text {field }}^{m-1}+\mathcal{O}_{\text {field }}^{m}}{2}\right\rangle_{p(c)}}{\left\langle\mathcal{O}_{\text {bath }}\right\rangle_{p(c)}},
$$

in terms of which the ground state energy is well approximated by (see Eq. 11)

$$
E_{M} \equiv E_{\text {ising }}+E_{\text {bond }}+N_{\text {site }} .
$$

In summary, we use the following formula as a QMC estimate of the Berry curvature,

$$
F_{s \phi}(M) \equiv \frac{-M N_{\text {bond }}(1-s)\left\langle\mathcal{O}_{y z}\right\rangle_{p(c)}}{2 E_{M}\left\langle\mathcal{O}_{\mathbb{1}}\right\rangle_{p(c)}}
$$

which becomes exact in the limit $M \rightarrow \infty$. 